\title{
Interprofessional Communication and Relationships in the Management of “Difficult to Treat” Depression: Perceptions of the Role of General Practitioners
}

\author{
Kay M. Jones*, Leon Piterman \\ Office of the Pro Vice-Chancellor, Peninsula Campus, Monash University, Frankston, Australia \\ Email: "kay.jones@monash.edu
}

Received 27 April 2015; accepted 1 June 2015; published 4 June 2015

Copyright (C) 2015 by authors and Scientific Research Publishing Inc.

This work is licensed under the Creative Commons Attribution International License (CC BY).

http://creativecommons.org/licenses/by/4.0/

(c) (i) Open Access

\begin{abstract}
Background: Team based care is an essential ingredient of chronic disease management including chronic mental illness. Effective health care teams include members who have defined, yet intersecting roles, where mutual respect characterises professional interaction and the patient's wellbeing is central. The aim was to explore the perception of psychologists, psychiatry registrars and psychiatrists with respect to GPs' role in managing difficult-to-treat-depression (DTTD). Methods: A previously developed semi-structured interview schedule comprising six questions was used. Thirty-two health professionals participated. Data were analysed using the Framework method. Findings: Four main themes emerged: 1) The team approach was important, particularly to ensure information accuracy and/or when responding to patient needs and pressures; 2) Referrals, usually generated by GPs can be a vehicle for other health professionals to provide advice to the GP; 3 ) Availability and accessibility often depended on health professionals work location and knowing how to navigate the system; 4) Limited availability of government funding impacts on patients' accessibility to health professionals. Discussion: Interprofessional relationships were described as paramount. Appropriate and timely referrals are integral to patient management, regardless of challenges. Ongoing challenges include program funding, workforce numbers and costs to patients. Improvement to mental health care access was noted, even for patients among relatively disadvantaged groups and those receiving Medicare Benefits Schedule-subsidised services. Conclusion: Despite adequate GP/specialist communication, the delivery of optimal team based care to patients with difficult-to-treat depression is compromised by lack of access to specialised services and inadequate funding.
\end{abstract}

*Corresponding author.

How to cite this paper: Jones, K.M. and Piterman, L. (2015) Interprofessional Communication and Relationships in the Management of "Difficult to Treat" Depression: Perceptions of the Role of General Practitioners. Open Journal of Psychiatry, 5, 260-266. http://dx.doi.org/10.4236/ojpsych.2015.53029 


\section{Keywords}

\section{Depression, Perceptions, Psychiatry}

\section{Background}

Team based care is an essential ingredient of chronic disease management [1] including the management of chronic or relapsing mental illness, major depression and difficult to treat depression (DTTD) falling into this category. Effective health care teams are those whose members have defined, yet intersecting roles, where mutual respect characterises professional interaction. In all cases, the patient's well-being should be at the centre of team based decision making and care.

While legal, ethical and professional requirements may vary, interpersonal relationships between general practitioners (GPs) and care team professionals are contextually and systematically determined [2]. Thus, many factors may influence the success, or otherwise, of inter-professional/multidisciplinary service provision including the structure of the care delivery pathway, funding models, practice culture, types of information, activities, and services or funding exchanges as well as the relationship governance in terms of accountability, professionalism, autonomy and power [3]. Although issues such as lack of time and inter-professional communication difficulties may arise [4]-[6], health professionals have reported beneficial changes in attitudes and knowledge as a result of experience gained from working with other professionals, understanding their roles and knowledge, communication and administrative systems [6].

Referrals are an important form of communication between the GP and other health professionals. They require a clear explanation of the problem with adequate patient history from the GP, a response outlining diagnosis and management, justification for the course of action from the care team professional/s; and the patient expects a clear explanation that describes the diagnosis, treatment and follow-up requirements. When this information is not provided, some or all involved in the process may become dissatisfied [7].

In Australia, team-based models of primary care have emerged in response to health system changes and challenges such as complex patient profiles, patient expectations and health system demands [8]. Overwhelmingly, the introduction by the Australian Government of the Better Access to Psychiatrists, Psychologists and General Practitioners (GPs) (the Better Access initiative) [9], has made services more accessible and more affordable for individuals who experience mental health disorders. GPs are providing more mental health services than in the past; patients now have access to psychological services that were previously less affordable and/or accessible, and there have been some changes to the way some psychiatrists provide care, including partnerships between the public and private psychiatric services [9]-[12]. In 2010-2011, \$6.9 billion was spent in Australia on mental health for national and state programs and initiatives [13].

Depending on the patient's needs, a care team may include a psychologist, psychiatry registrar and/or psychiatrist, social worker, counsellor to whom patients may have been referred by a GP. Apart from work previously published by this team [14]-[16], no literature could be found describing care team members' thoughts about GPs' role in the management of patients diagnosed with DTTD, or about care team members' interactions.

The aim of this qualitative research was to explore the perception of psychologists, psychiatry registrars and psychiatrists with respect to GPs' role in managing DTTD.

\section{Methods}

For the purpose of this paper, the description of DTTD as "most often conceptualized in terms of repeated failures to ameliorate depressive symptoms" is used [17].

\subsection{Sample Recruitment}

A convenience sample was recruited via emails which were forwarded to GPs in 2011, psychologists and psychiatry registrars in mid-2012, and psychiatrists early in 2014. All had links to Monash University, and/or the Monash Medical Centre (a public hospital in Melbourne, Australia). When potential participants responded and agreed to participate, they provided and/or confirmed their contact details (email) to the research team for the purpose of the research team advising time, date and venue for the focus group [18] [19]. 


\subsection{Data Collection and Analysis}

A semi-structured interview schedule comprising six headings was developed for interviews and/or focus groups with GPs [14], psychologists [16] psychiatry registrars [15] and psychiatrists.

Data were collected in Melbourne in late 2011(GPs), mid 2012 (psychologists and psychiatry registrars) and early 2014 (psychiatrists). The interviews lasted approximately thirty-to-forty five minutes; the focus groups lasted approximately ninety minutes. All were audio-taped and transcribed verbatim. Apart from gender, profession and workplace experience (public/private, urban/rural) no other demographic data were collected.

Data were analysed using the Framework Method [20] to understand participants' perspectives. Data were analysed manually and independently by the investigators. When there was a difference of opinion, the issues were discussed and agreement reached [20]. Findings, including discussion are reported under the interview schedule's six headings. Comments are reported for GPs as GPFG 1-5 and GPIV 1-5, for psychologists as P1-P7, for psychiatry registrars as PR 1-10, and for psychiatrists as PS1-5.

Ethics approval to conduct the study was obtained from Monash University Human Research Ethics Committee (MUHREC) and Monash Health.

\section{Findings}

Findings are reported under the four main themes that emerged from the data.

\subsection{Participants}

\begin{tabular}{ccccc}
\hline Group & Females & Males & Experience in public (Pu)/private (Pr) & Experience in urban (U)/rural (R) \\
\hline GPs & 3 & 7 & $10(\mathrm{Pu} \& \mathrm{Pr})$ & $8(\mathrm{U}) 2(\mathrm{R})$ \\
Psychologists & 5 & 2 & $7(\mathrm{Pu} \& \mathrm{Pr})$ & $5(\mathrm{U}) 2(\mathrm{R})$ \\
Psychiatry registrars & 6 & 4 & $10(\mathrm{Pu})$ & $10(\mathrm{U})$ \\
Psychiatrists & 0 & 5 & $5(\mathrm{Pu} \& \mathrm{Pr})$ & $5(\mathrm{U})$ \\
Total & $\mathbf{1 4}$ & $\mathbf{1 8}$ & & \\
\hline
\end{tabular}

\section{1) Significance of relationship between the GP and other health professionals}

"The relationship between the GP and other health professionals is vital, and for the patient: it's a matter of the GP finding the right professional for the patient" (GPIV2).

Psychologists reported working in both the public and private sections of the health system in Australia and receive referrals and patient histories from GPs and other health professionals who may be involved in the patient's primary care. When clarification was needed, the most important starting point for the psychologist was usually the GP, for example, contacting the GP to check if the GP has tried different medications, because the patient may be treatment resistant was considered important. However, conflicting with the importance of the interaction between professionals was their availability:

"GPs can't always be readily accessed, particularly those who work in multiple clinics, thus setting up meetings with the GP, psychologist and psychiatrist can be difficult" (P5).

The psychiatry registrars described GPs as knowing the patients and building a relationship with them, and that GPs are holistic practitioners who see a range of people, have a sense of what else may be going on, can often clearly say what's happening and who are often really good for connectedness with the patient and it's that connectedness and that can help. But again:

"Time constraints and busy schedules were identified as difficulties for some GPs" (PR6).

Psychiatry registrars revealed insights into the difficulties experienced by GPs. They felt that GPs may be challenged by some patients' behaviour, such as those at risk of suicide and those who make demands for prescription drugs or referrals, adding pressure for the GP either because of time constraints or the GP not wanting 
to be seen to be doing nothing. Subsequently, for GPs or any health professional in a care team, patient pressures and lack of time can impact on relationships with patients and interactions with other health professionals because:

"More often than not, by the time the patient gets to the psychiatry registrar's clinic, private or public, they've already been treated by the GP and sometimes have also seen a psychologist" (PR2).

Psychiatrists also described the importance of the team approach:

"We work in conjunction with the GP and the team might include a psychologist or a social worker or other allied health professionals such as an occupational therapist" (PS4).

\section{2) Referring and management}

"Referrals are usually from GPs to care-team health professionals with GPs often receiving regular feedback from their patients about the specialists to whom they were referred" (GPIV5).

From the psychologists perspective co-morbidities might impede the psychologist's ability to engage the patient, particularly patients with progressive illness, progressive degenerative illness such as early dementia or Parkinsons or MS where there's a cognitive element that might be contributing biologically to a lack of motivation that may impact on treatment. While psychologists may refer a patient on to a psychiatrist, generally they would go back to the referrer who is usually the GP with a recommendation for the particular patient. Psychologists also noted that in some instances, referrals need to be made in a specific way, for example via a mental health care plan in order to access a government subsidised service, and to work collaboratively and support GPs:

"Some psychologists may write to the GP advising what services are available and how the GP can refer, because some GPs have no idea about these services and don't have time, so the psychologist's input makes it easier for the GP to refer" (P7).

"It's about finding the balance of being able to give the GP the information, or pointing them in the direction and making a recommendation" (P4, P5).

From the psychiatry registrars' perspective, it was the referring GP who knows and builds a relationship with the patient and understands their life, their paths, their psycho-social stressors and the way the patient relates to others, including the GP:

"I find that if I have been asked to see somebody by another member of the medical team and then I call the GP, the GP can often very clearly say what and why this is happening" (PR5).

"But, by virtue of being a psychiatry registrar in a public hospital, we're the second line already" (PR10).

In addition to receiving referrals from GPs, psychiatrists may refer to other health professionals including psychologists, occupational therapists and social workers in public as well as private practice. Hence they are:

"Inextricably linked to the GP through the referral and health system" (PS5).

\section{3) Availability and accessibility of health professionals}

“Deinstitutionalisation of mental health patients in Australia in the late 1980's and 1990's resulted in significant numbers of patients with a mental health diagnosis being placed in the community without the resources there to support them: this resulted in some hard times in the 1990's in just trying to pick up the pieces there for a while" (GPIV1).

More recently, while GPs endeavour to manage patients via access to primary mental health teams to provide support and assist with patients' treatment, availability and accessibility can depend on whether the health professional is working in the public or private sector of the health system in Australia. Currently, not all patients are referred to psychiatrists for a range of reasons:

"Including lack of availability and difficulty in getting an appointment, whether in the public or private sectors and particularly in the rural areas" (GPIV 1, GPIV4).

Psychologists also found difficulty accessing various services, but acknowledged that mental health support and services have been more accessible in recent years because of the Medicare rebate available via the "Better 
Access" initiative. With more psychologists and social workers working in the community there are significantly more health professionals to access for the patient management and treatment. Nonetheless challenges remain within the systems, for example:

"There's a certain language that needs to be used with triage to either speed up the referral or to ensure that referral's heard and I think that's still a difficulty" (P3).

"Sometimes you have to be pretty assertive and that's pretty hard" (P1).

While most services have clear admission criteria requirements, sometimes stigmatising can preclude some patients such as those with co-morbidity or substance abuse. Thus, regardless the best endeavours and support of all health professionals involved:

"Making those referrals is actually more difficult because the person's not particularly attractive for the accepting service to pick up. That still happens, yep" (P5).

From the psychiatry registrars' perspective it's not about availability and accessibility because, for them, some of the social issues can be delegated to the social workers and:

“Being psychiatry registrars in a public hospital we're second line already" (PR3).

Psychiatrists raised the issue of containment:

"Rather than availability and accessibility or management options, sometimes for psychotic patients it's about containment, whether it's in the community or in a patient unit using medication" (PS3).

\section{4) Funding/financial issues}

Sustainable government supported funding models are essential for effective care delivery.

Changes in government funding and programs in Australia have impacted on initiatives delivered in the community and on the health professionals involved:

"Our general practice was part of a primary health team initiative (the Better Access program), a psychologist came to the practice once every three to four weeks for around two years, but the funding and program closed and subsequently, the service discontinued" (GPIV 1).

Psychologists raised similar issues including queries around government funded consultations with psychologists and psychiatrists, and the limits on the number of visits under the Medicare "Better Access" program:

“There's always some patients who can't afford the cost, which can result in patients deciding to stop seeing the psychologist and probably end up back at the GP” (P3, P6, P7).

Psychiatry registrars and psychiatrists expressed concerns about the nexus between hospital and community based treatment. If government subsidised community based services are not available, discharge planning may be delayed:

"In some suburbs it is more difficult again because of patients' limited resources which would factor into decision making about discharging a patient" (PR5).

"Despite funding for various electronic forms of communication, such as psychiatry on line or telephone counselling service, which are government supported, few utilise these options” (GPFG3, GPFG5).

\subsection{Summary of Key Findings}

Four main themes emerged:

1) The team approach is paramount, particularly to ensure information is accurate and/or when responding to patient needs and pressures.

2) Referrals are usually generated by GPs; they can be a vehicle for other health professionals to provide advice to the GP, and may need to be made in a specific way in order to access a particular service.

3) Availability and accessibility often depended on where the health professional works (public or private), knowing how to navigate the system, and being aware that some patients may be stigmatised and some may need containment. 
4) Changes in government funding and programs, particularly the limited availability of "bulk-billing” impacts on patients' accessibility to health professionals, resulting in patients some being treated by the GP only.

\section{Discussion}

This paper is one of few that describes the thoughts of health professionals about GPs' role in the management of patients diagnosed with difficult-to-treat depression or about the care team member's interactions [14]-[16].

As previously reported [5] [8] [9] [11], participants in this research also identified the GP as usually being the most accessible medical resource in the community and the first point of professional contact for many people seeking help with mental health problems. Similarly, the interprofessional relationship between the GP and other health professionals was described as paramount, particularly when multiple health professionals are involved in a patients' management.

In the past, access to mental health services was limited due to workforce shortage, uneven geographical distribution and access barriers including affordability [5] [10]. For example, prior to June 2008, only GPs could refer patients to the "Access to Allied Psychological Services (ATAPS)" (a component of the Better Outcomes initiative) but in June 2008, policy changes included the introduction of the Suicide prevention program and extending "who" could refer to ATAPS to include mental health services and psychiatrists [11]. Outcomes described in the "Better Access" initiative evaluations [9]-[11] [13] were reflected in participants responses, particularly the provision of support for GPs, referral pathways for appropriate treatment by psychologists, psychiatrists and other appropriately trained mental health professionals, the benefits of the team approach and improving affordable access for patients to services.

General consensus was that appropriate and timely referral was integral to patient management, regardless of challenges such as patients not responding to psychological or psychiatric management [14]. Specific issues identified as impacting on referral process included accessibility and communication. Previous research suggests that communication can be improved by utilising formal and informal meetings [12], improving letter writing skills [4] particularly for referrals [7] and utilising networking and interprofessional education which may help these professionals work more effectively in team based care [8] [9] [12].

All noted that the availability and accessibility of GPs, psychologists, psychiatry registrars and psychiatrists, particularly in rural areas, were described as ongoing challenges [5] [10] [13] [14]. Tied to availability and accessibility, are the costs for the patient and program funding by the government, but despite the gap between fee and rebate, improvement to access of mental health care was noted, even for patients among relatively disadvantaged groups in the community and those receiving Medicare Benefits Schedule-subsidised services, including bulk-billing (government supported fees) [9] [10] [13].

\section{Conclusion}

While the generalisability of this study may be limited because of the small number of participants, this study is the first to contribute to gaining some insight into care team members' thoughts about the GPs' role in the management of patients diagnosed with DTTD, or about care team members' interactions. Psychiatrists, psychiatry registrars and psychologists all acknowledged the pivotal role of GPs in managing mental illness. Whilst in relative terms the Australian health care system is working, significant constraints related to accessibility and funding to support specialized care and team based care compromise care for patients with mental illness in particular those with major depression or DTTD. Regardless of the level and content of interprofessional communication and relationships, more funding is needed to make interprofessional communication more meaningful and effective for the fee-for-service health care delivery system in Australia.

\section{Conflict of Interest}

None.

\section{References}

[1] Wagner, E.H. (1998) Chronic Disease Management: What Will It Take to Improve Care for Chronic Illness? Effective Clinical Practice, 1, 2-4.

[2] Piterman, L. and Koritsas, S. (2005) Part 1 General Practitioner-Specialist Relationship. Internal Medicine Journal, 


\section{5, 430-434. http://dx.doi.org/10.1111/j.1445-5994.2005.00855.x}

[3] Bowers, E.J. (2010) How Does Teamwork Support GPs and Allied Health Professionals to Work Together? PHCRIS.

[4] Sain, K., Shah, N. and Hasan, S. (2012) GPs' Views on the Content and Quality of Psychiatrists' Clinic Letters. Progress in Neurology and Psychiatry, 16, 6-10. http://dx.doi.org/10.1002/pnp.223

[5] (2006) Senate Select Committee Mental Health: A National Approach to Mental Health—From Crisis to Community. Final Report. Senate Select Committee, Commonwealth of Australia, Canberra.

[6] Goldman, J., Meuser, J., Rogers, J., Lawrie, L. and Reeves, S. (2010) Interprofessional Collabaoration in Family Health Teams. Canadian Family Physician, 56, e368-e374.

[7] Piterman, L. and Koritsas, S. (2005) Part II General Practitioner-Specialist Referral Process. Internal Medicine Journal, 35, 491-496. http://dx.doi.org/10.1111/j.1445-5994.2005.00860.x

[8] Nacarella, L., Greenstock, L.N. and Brooks, P.M. (2012) A Framework to Support Team-Based Models of Primary Care within the Australian Health Care System. MJA Open, 1, 22-25. http://dx.doi.org/10.5694/mjao12.10069

[9] Pirkis, J., Harris, M., Hall, W. and Ftanou, M. (2011) Evaluation of the Better Access to Psychiatrists, Psychologists and General Practitioners through the Medicare Benefits Schedule Initiative. Summative Evaluation, Final Report. Centre for Health Policy, Programs and Economics, The University of Melbourne.

[10] (2010) Department of Health and Ageing: Evaluation of the Better Access Initiative Final Report. Department of Health and Ageing.

[11] Fletcher, J., King, K., Bassilios, B., Reifels, L., Blashki, G., Burgess, P. and Pirkis, J. (2012) Evaluating the Access to Allied Psychological Services (ATAPS) Program. Centre for Health Policy Programs and Economics, University of Melbourne. https://ataps-mds.com/site/assets/.../19th_interim_evaluation_report.pdf

[12] Yung, A., Gill, L., Somerville, E., Dowling, B., Simon, K., Pirkis, J., Livingston, J., Schweitzer, I., Tanaghow, A., Herrman, H., et al. (2005) Public and Private Psychiatry: Can They Work Together and Is It Worth the Effort? Australian and New Zealand Journal of Psychiatry, 39, 67-73. http://dx.doi.org/10.1080/j.1440-1614.2005.01511.x

[13] Department of Health and Ageing (2013) National Mental Health Report 2013: Tracking Progress of Mental Health Reform in Australia 1993-2011. Commonwealth of Australia, Canberra.

[14] Jones, K.M., Castle, D.J. and Piterman, L. (2012) Difficult-to-Treat-Depression: What Do General Practitioners Think? MJA Open, 1, 6-8. http://dx.doi.org/10.5694/mjao12.10566

[15] Jones, K.M. and Piterman, L. (2014) Difficult-to-Treat-Depression and GPs’ Role: Perceptions of Psychiatry Registrars. Open Journal of Psychiatry, 4, 301-308. http://dx.doi.org/10.4236/ojpsych.2014.44037

[16] Jones, K.M. and Piterman, L. (2014) Difficult-to-Treat-Depression and GP’s Role: Perceptions of Psychologists. Open Journal of Psychiatry, 5, 31-38. http://dx.doi.org/10.4236/ojpsych.2015.51005

[17] Grote, N.K. and Frank, E. (2003) Difficult-to-Treat Depression: The Role of Contexts and Comorbidities. Society of Biological Psychiatry, 53, 660-670. http://dx.doi.org/10.1016/S0006-3223(03)00006-4

[18] Liamputtong, P. and Ezzey, D. (2005) Qualitataive Research Methods. Oxford University Press, Melbourne.

[19] Polgar, S. and Thomas, S. (2005) Introduction to Research in the Health Sciences. Elsevier Churchill Livingstone, Sydney.

[20] Ritchie, J. and Spencer, L. (1994) Qualitative Data Analysis for Applied Policy Research. In: Bryman, A. and Burgess, B., Eds., Analyzing Qualitative Data, Routledge, London. http://dx.doi.org/10.4324/9780203413081_chapter_9 